\title{
Polycrystalline CVD Diamonds for the Beam Calorimeter of the ILC
}

\author{
Christian Grah, Konstantin Afanaciev, Igor Emeliantchik, Ulrich Harder, Hans Henschel, Alexandr Ignatenko, \\ Ekaterina Kouznetsova, Wolfgang Lange, Wolfgang Lohmann, Martin Ohlerich, and Ringo Schmidt
}

\begin{abstract}
Polycrystalline artificial diamond produced by chemical vapor deposition (pCVD) is a possible sensor material for the beam calorimeter of the ILC. The requirements are linearity over a large range of flux and radiation hardness against a total ionizing dose of several MGy per year of operation. A hadron test beam at the CERN PS was used to study the linearity of the response of pCVD sensors. An electron test beam at the S-DALINAC was used to measure the charge collection distance (CCD) as a function of the absorbed dose up to several MGy. Current-voltage characteristics of these sensors were measured before and after the irradiation as well as the dependence of the CCD on the applied electric field before and after the irradiation.
\end{abstract}

Index Terms-BeamCal, charge collection distance, CVD diamond sensor, FCAL, forward calorimetry, ILC, irradiation, pumping, radiation hardness.

\section{INTRODUCTION}

$\mathbf{T}$ HE International Linear Collider (ILC) will be the next generation facility for high energy physics after the Large Hadron Collider. The ILC will be an electron-positron collider with a tunable center-of-mass energy up to $500 \mathrm{GeV}$, with a possible upgrade to $1 \mathrm{TeV}$. The luminosity required from the physics program will be up to $10^{34} \mathrm{~cm}^{-2} \mathrm{~s}^{-1}$. Due to the high particle density the emission of beamstrahlung photons occurs during the collision of bunches. These photons leave the detectors at the ILC through the beam pipe but they also partly convert to electron-positron pairs. A fraction of these pairs is deflected to larger polar angles and constitutes the major background the detectors have to deal with. The forward region of the ILC detectors is affected the most [1]. The innermost calorimeter, BeamCal, positioned about $3.6 \mathrm{~m}$ from the interaction point, IP, is hit by a large amount of the pairs from

Manuscript received March 28, 2008; revised November 22, 2008 and December 18, 2008. Current version published April 08, 2009. This work is supported in part by the Commission of the European Communities under the 6th Framework Programme "Structuring the European Research Area," Contract RII3-026126, by the German Federal Ministry for Education and Research, and by a Björn Wiik Research Scholarship of the World Laboratory.

C. Grah, U. Harder, H. Henschel, W. Lange, and W. Lohmann are with the FCAL Group, DESY, Zeuthen, Germany (e-mail: christian.grah@desy.de; ulrich.harder@desy.de; hans.henschel@desy.de; wolfgang.lange@desy.de; wolfgang.lohmann@desy.de).

K. Afanaciev, I. Emeliantchik, and A. Ignatenko are with NC PHEP, Belarusian State University, Minsk, Belarus (e-mail: konstantin.afanaciev@desy.de; igor.emeliantchik@desy.de; alexandr.ignatenko@desy.de).

E. Kouznetsova was with the FCAL Group, DESY, Zeuthen, Germany. He is now with INFN Rome c/o Physics Department, Sapienza University, Rome, Italy (e-mail: ekaterina.kouznetsova@desy.de).

M. Ohlerich and R. Schmidt are with the FCAL Group, DESY, Zeuthen, Germany, and the Technical University of Cottbus, Cottbus, Germany (e-mail: martin.ohlerich@desy.de; ringo.schmidt@desy.de).

Digital Object Identifier 10.1109/TNS.2009.2013853

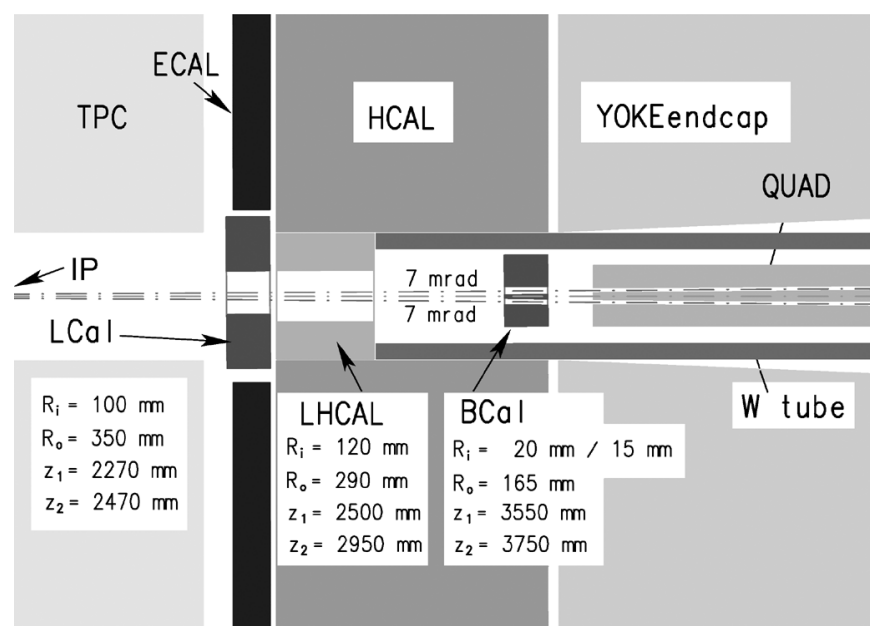

Fig. 1. Forward region of the Large Detector Concept (LDC) for a beam crossing angle of $14 \mathrm{mrad}$. BCal corresponds to the BeamCal and is positioned within the tungsten support tube, directly in front of the last quadrupole magnet (QUAD) of the beam delivery system. Also shown are the luminometer (LCal), the calorimeter systems ECAL and HCAL in the end cap, the time projection chamber (TPC), the low angle hadron calorimeter (LHCAL) and the instrumented return yoke for the magnetic field (YOKEendcap).

beamstrahlung. BeamCal is designed as an electromagnetic sandwich calorimeter situated directly in front of the final focus magnets and adjacent to the beam pipe. The minimal polar angle covered by BeamCal is $5 \mathrm{mrad}$ and it reaches up to $45 \mathrm{mrad}$ in the current layout for the Large Detector Concept, LDC [2], which is shown in Fig. 1. BeamCal comprises 30 layers of tungsten as the absorber material, each of a thickness of $3.5 \mathrm{~mm}$ which corresponds to one radiation length $\mathrm{X}_{0}$. Thirty layers of sensors are interspersed between the absorber planes and measure the electromagnetic cascade. The overall shape of BeamCal is cylindrical with an outer radius of up to $16.5 \mathrm{~cm}$ and additional space for the readout electronics.

The signals from beamstrahlung pairs in the sensor layers are used to obtain information about the initial collision and to provide a feedback signal to the machine steering systems. Certain physics scenarios require a sensitivity to single high energy electrons in the acceptance range of BeamCal. It is therefore designed as a very compact calorimeter with high granularity in the sensors. The Molière radius, $\mathrm{R}_{\mathrm{M}}$, will be about $1 \mathrm{~cm}$ and the anticipated segment size of the sensors is between 0.5 and $0.8 \mathrm{R}_{\mathrm{M}}$. The ability to detect the energy deposition from single high energetic particles on top of the background from beamstrahlung pairs relies on the usage of sensors with a dynamic range of about $10^{3}$. 


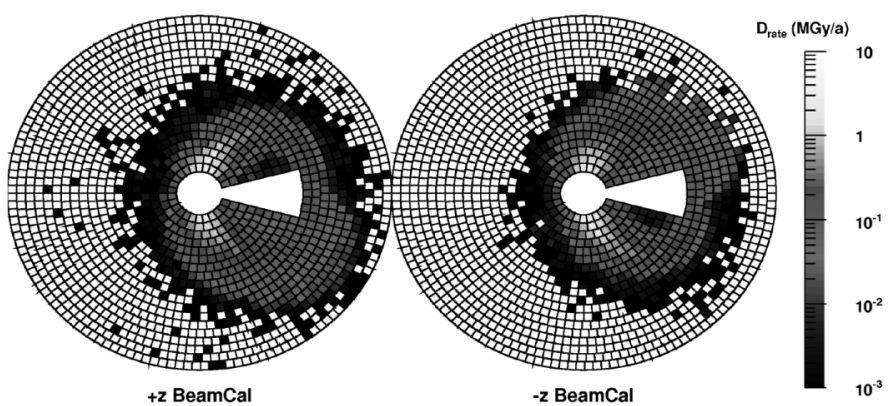

Fig. 2. Expected annual dose rate for a BeamCal sensor layer. The layer shown is the one with maximum energy deposition (after $6 \mathrm{X}_{0}$ ). This case is for nominal beam parameters and a magnetic field of $4 \mathrm{~T}$ parallel to the incoming beam. The beam crossing angle leads to a not instrumented area around the incoming beam pipe. The detector is centered on the outgoing beam pipe. Note that at the outer radii a white fill color denotes a dose rate of less than $1 \mathrm{kGy}$ per year.

The Total Ionizing Dose, TID, absorbed in the sensor segments can accumulate to several MGy per year of operation. Fig. 2 shows the expected dose per year in a sensor layer of BeamCal as a result of a Geant4 [3] based simulation of the forward region. The expected dose per year is shown after $6 \mathrm{X}_{0}$ of absorber which corresponds to the sensor layer with the maximal energy deposition. A permanent operation of the machine at nominal beam parameters [4] is assumed for the calculation. The maximal expected dose from this simulation is 3.6 MGy per year in the sensor cells adjacent to the beam pipe. The use of radiation hard sensors is therefore mandatory. The shape of the energy deposition and the absolute value is strongly dependent on the parameters of the colliding beams and the magnetic field configuration in the detector. Including a safety factor it is reasonable to set the benchmark for the radiation hardness of the sensors to 10 MGy per year of operation.

Diamonds grown by chemical vapor deposition, CVD, are a potential sensor material. This material has been investigated for its radiation hardness in the context of its use as an alternative sensor material for the silicon tracking detectors in the LHC experiments as described in [5] and [6] and references quoted therein. These studies investigate the radiation damage by proton or neutron fluence, which are expected to be relative low in our application, while the TID is a factor of 1000 larger than e.g. the requirements for the LHC tracking detectors. Radiation hardness studies with photons from synchrotron radiation and Cobalt decays are described in [7].

\section{CVD DIAMONDS}

Polycrystalline CVD diamonds have become available in wafer sizes and in the quantities necessary to build detector systems. The principle of operation is similar to that of silicon detectors. A metallization is deposited on both sides of the material to allow the application of an electric field to the sensor. An ionizing particle traversing the sensors creates electron-hole pairs along its path. The drift of these charge carriers in the electric field induces a measurable signal in the electrodes, which can be detected by adequate readout electronics. One property of the diamond material is its large band gap of $5.45 \mathrm{eV}$. The large band gap-for comparison the band gap of silicon is $1.12 \mathrm{eV}$-leads to two important effects in our application: the first is that the dark current in the material is very low and the second is that the mean ionization energy necessary to create an electron hole pair by ionizing radiation is relatively large. The mean ionization energy is $13.1 \mathrm{eV}$ [8], considerably higher than the one for silicon $(3.63 \mathrm{eV})$. This is partly compensated by the higher density of diamond, which leads to a higher energy deposition by ionization. The signal expected from a minimum ionizing particle, MIP, in an ideal diamond sensor is therefore significantly smaller than for silicon, about 3600 compared to 9200 eh-pairs per $100 \mu \mathrm{m}$ [5]. Due to charge trapping at grain boundaries, the charge collection efficiency for polycrystalline material is usually significantly lower than $100 \%$. The signal in pCVD diamond due to a MIP is often expressed in terms of the Charge Collection Distance, CCD. The CCD is the mean distance a charge carrier drifts in the material

$$
C C D=F \times \Sigma_{i=e, h}\left(\tau_{i} \times \mu_{i}\right)
$$

where $\tau_{i}$ is the average lifetime of an electron or hole before it gets trapped, $\mu_{i}$ is the corresponding mobility and $\mathrm{F}$ the applied electric field. The CCD can also be interpreted as

$$
C C D=\varepsilon \times d=\frac{Q_{e x p}}{Q_{\text {theo }}} \times d
$$

where $\varepsilon$ is the charge collection efficiency of the sensor with the thickness $\mathrm{d}$, which is determined by the ratio of the measured charged $Q_{\text {exp }}$ and the expected charge $Q_{\text {theo }}$. The expected charge $Q_{\text {theo }}$ is 36 eh-pairs per $\mu \mathrm{m}$ of traversed material [9].

These issues are important for the detection of individual MIPs as it is required for tracking detectors. However for a calorimeter the absolute signal size of a MIP is usually of secondary importance, due to the high number of particles within the electromagnetic cascade.

Major concerns in our application are the linearity, that is the dependence of the signal on the particle flux through the sensor and the understanding of the degradation of the sensor response as a function of the absorbed dose. We investigate polycrystalline diamond samples from two manufacturers: Element Six Ltd., E6, UK, and the Fraunhofer Institute for Applied Solid-State Physics, IAF, Germany. The metallization of the samples is a Ti-Pt-Au three layer metallization of one $\mathrm{cm}^{2}$ size.

The CCD is measured in a setup as shown in Fig. 3 using a ${ }^{90} \mathrm{Sr}$ source. The source emits electrons with an energy up to $2.3 \mathrm{MeV}$. Particles traversing the sensor and generating a signal in the scintillator originate from the high energy tail of the spectrum. They can be regarded as being MIP-like, with an expected signal of 36 electron-hole pairs per $\mu \mathrm{m}$ of traversed diamond material. The signal of the sensor is amplified and shaped (charge sensitive preamplifier and additional shaper, both shown as 'preamplifier' in Fig. 3) and then digitized using a charge sensitive Analog to Digital Converter, ADC.

\section{LINEARITY TEST OF PCVD DIAMOND}

The linearity test of polycrystalline CVD diamonds has been carried out at the Proton Synchrotron facility, PS, of the European Organization for Nuclear Research, CERN. The PS pro- 


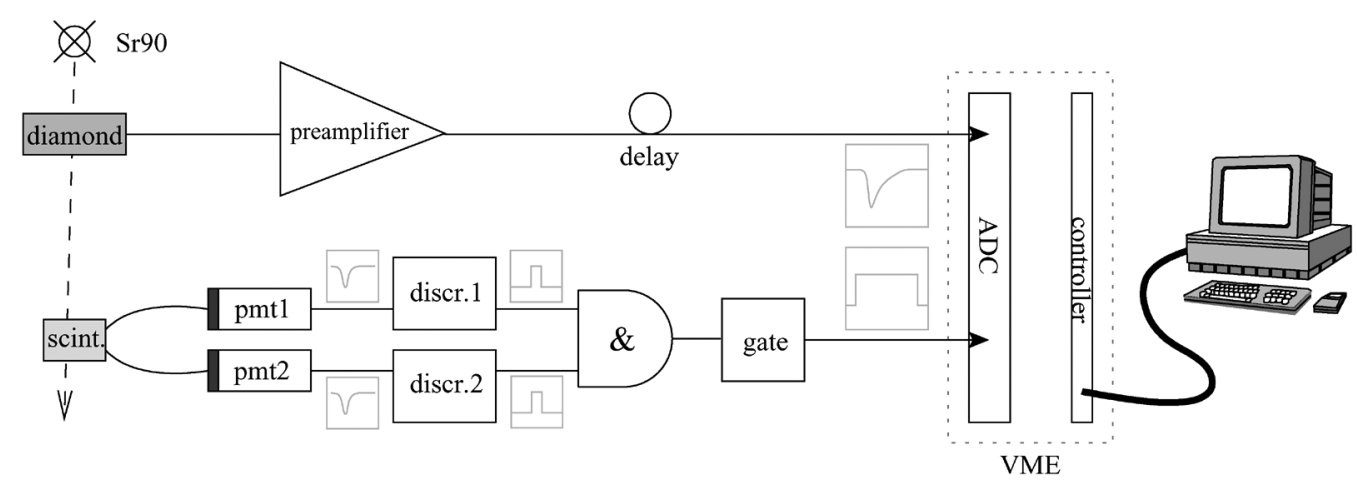

Fig. 3. The setup for spectroscopic measurements using ${ }^{90} \mathrm{Sr}$ as particle emitter. A charge sensitive preamplifier and an ADC is used to measure and digitize the signal of the sensor. A scintillator is used to generate a trigger signal for MIP-like particles.

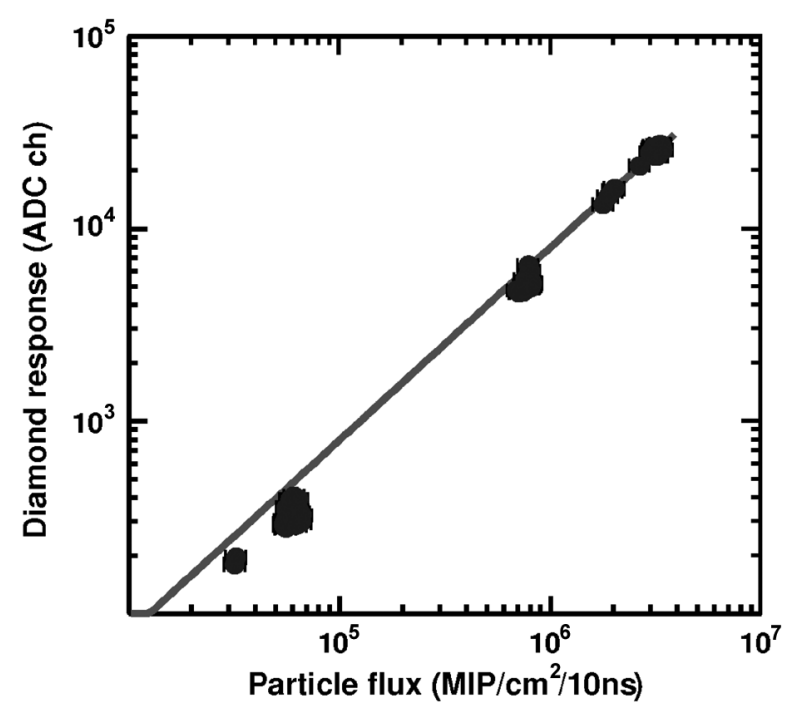

Fig. 4. The response of polycrystalline diamond sensors from E6 as a function of the particle flux in units of the number of particles per $\mathrm{cm}^{2}$ per $10 \mathrm{~ns}$. The bias voltage applied to the sensors was $500 \mathrm{~V}$. (Sample E6\#a, $500 \mu \mathrm{m}$ thick, $0.8 \mathrm{~V} / \mu \mathrm{m})$.

vides a $4 \mathrm{GeV}$ hadron beam of variable intensity in a $10 \mathrm{~ns}$ pulse. This is ideal to study the response of the sensors as a function of the particle flux.

The Device Under Test, DUT, is in this case directly connected to a charge sensitive Analog to Digital Converter, ADC. A scintillator is placed behind the DUT, which is read out using wavelength-shifting fibers connected to two photo-multipliers. Two photo-multipliers with different amplification are to monitor the beam intensity over a sufficiently large range. Their signals are digitized in ADCs and used in a coincidence to generate a trigger signal. The absolute measurement of the particle flux was obtained by the CMS BCM group [10] using thermoluminescence dosimetry. The combination of the two measurements allows the reconstruction of the actual flux per pulse.

In Fig. 4 and Fig. 5 the response of sensors as a function of the particle flux is shown separately for samples from the two manufacturers. The response is shown in ADC counts. Albeit the sensors are produced by different manufacturers using similar technologies, the variations between the manufacturing processes cause differences in the response to ionising particles. The data is fitted with a straight line. Additionally the response

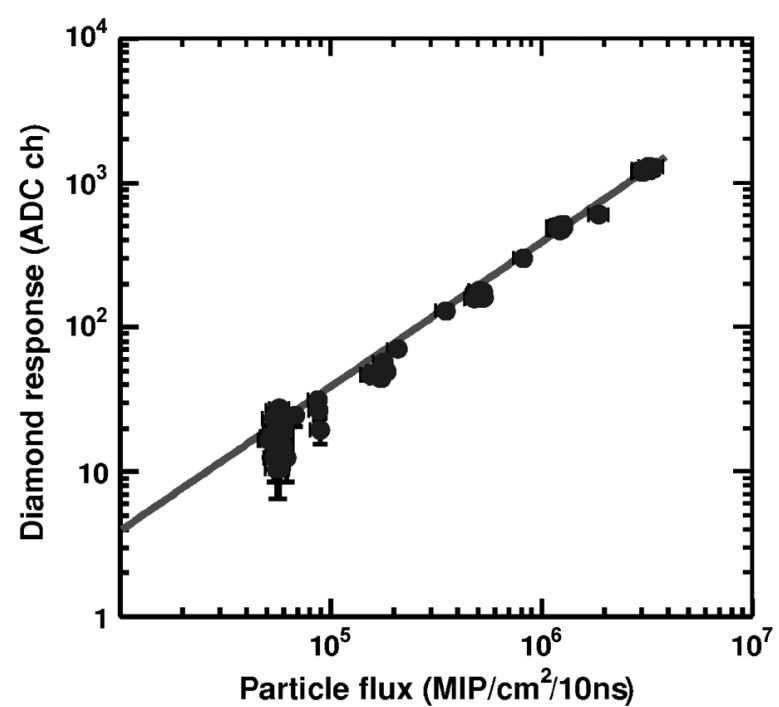

Fig. 5. The response of polycrystalline diamond sensors from IAF as a function of the particle flux in units of the number of particles per $\mathrm{cm}^{2}$ per $10 \mathrm{~ns}$. The bias voltage applied to the sensors was $500 \mathrm{~V}$. (Sample IAF\#a, $200 \mu \mathrm{m}$ thick, $2.0 \mathrm{~V} / \mu \mathrm{m})$.

of the sensor to single particles is included in the fit. These two data points are not shown in the plots to increase the visibility of the data points obtained by the test beam measurement [11].

The deviation from the straight line fit is less than $30 \%$ for both sensors. Deviations from the linear behaviour on this level of precision are explained by the limited precision of the flux reconstruction.

\section{IRRADIATION OF PCVD DIAMOND WITH ELECTRONS}

The energy of the particles originating from beamstrahlung pairs and depositing energy in the sensors of the BeamCal varies over a wide range as shown in Fig. 6. This spectrum is obtained from a GEANT4 based simulation of BeamCal. The spectrum shows that a large fraction of particles is of energies in the $\mathrm{MeV}$ range. A suitable accelerator for irradiation studies at these energies is the Superconducting Darmstadt Linear Accelerator, S-DALINAC, which provides an electron beam over a wide range of intensities and a beam energy between 10 and $130 \mathrm{MeV}$. This facility of the Technical University of Darmstadt, Germany, was used in summer 2006 to irradiate polycrys- 


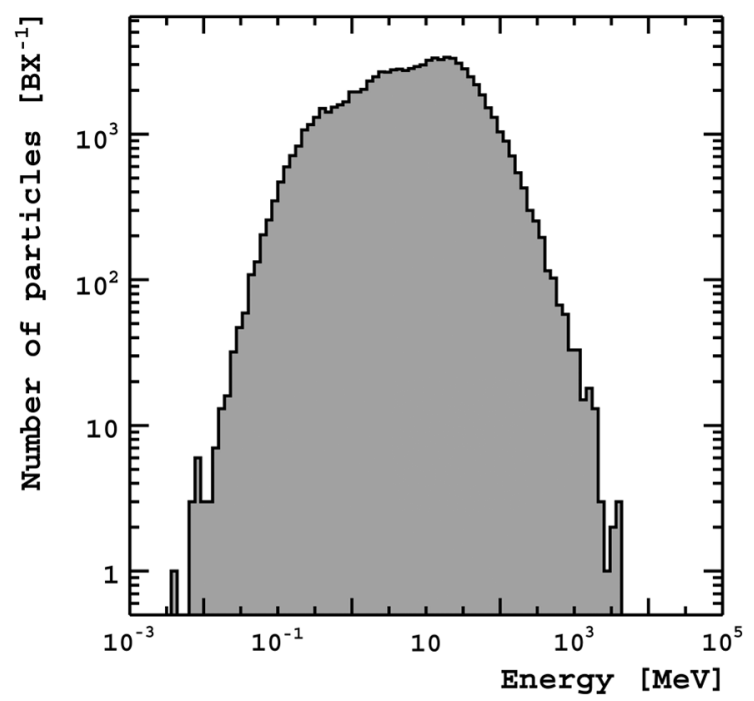

Fig. 6. The spectrum of particles, which deposit energy in the BeamCal sensor layer. The considered sensor is placed after 6 layers of absorber. The simulation was done for one bunch crossing at nominal beam settings.

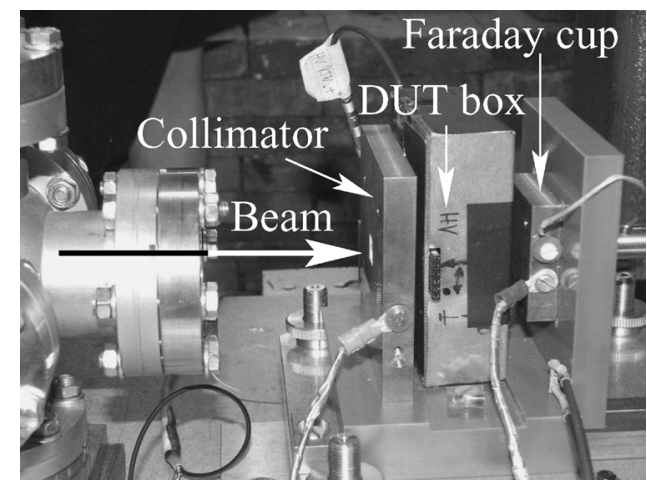

Fig. 7. Irradiation setup. The electron beam arrives from the left and is collimated to the sensitive area of the DUT by a copper collimator. The collimated beam size and sensor size match each other. The DUT is situated in a light tight box, with connectors for the bias voltage supply and the current monitoring. The beam current is measured by stopping the beam using a Faraday cup made from copper.

talline CVD diamond samples at a beam energy of $10 \mathrm{MeV}$ and up to a TID of several MGy.

One of the central aspects of the irradiation was to keep the sensor or device under test, DUT, under bias voltage during the measurement. Two setups were used during this study: a spectroscopic setup to determine the response from MIPs as described above and an irradiation setup as shown in Fig. 7 for exposing the DUT to the beam. A collimator and a Faraday cup are used to determine the electron current crossing the sensor.

The determination of the absorbed dose assumes a uniform distribution of the beam intensity over the DUT area. The average deposited energy per incident particle and a correction factor for the fraction of particles not crossing the sensor but measured in the Faraday cup are obtained from a GEANT4 simulation of the setup.

\section{A. Behavior During Irradiation}

In Fig. 8 and Fig. 9 the results are shown for the irradiation of diamond sensors from the two different manufacturers. We

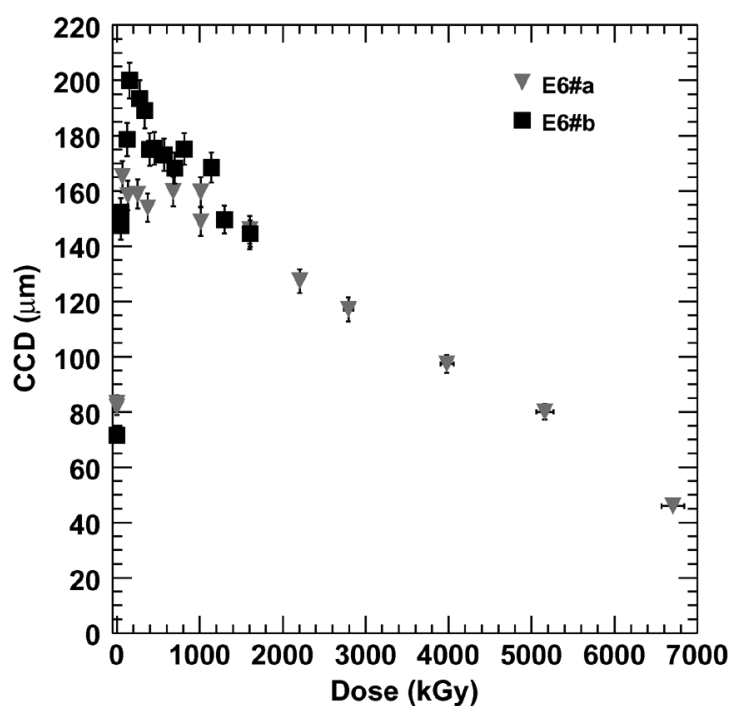

Fig. 8. The response of polycrystalline diamond sensors from E6 as a function of the absorbed dose from $10 \mathrm{MeV}$ electrons. The bias voltage applied to the sensors was $400 \mathrm{~V}$. (E6 samples, $500 \mu \mathrm{m}$ thick, $0.8 \mathrm{~V} / \mu \mathrm{m}$ ).

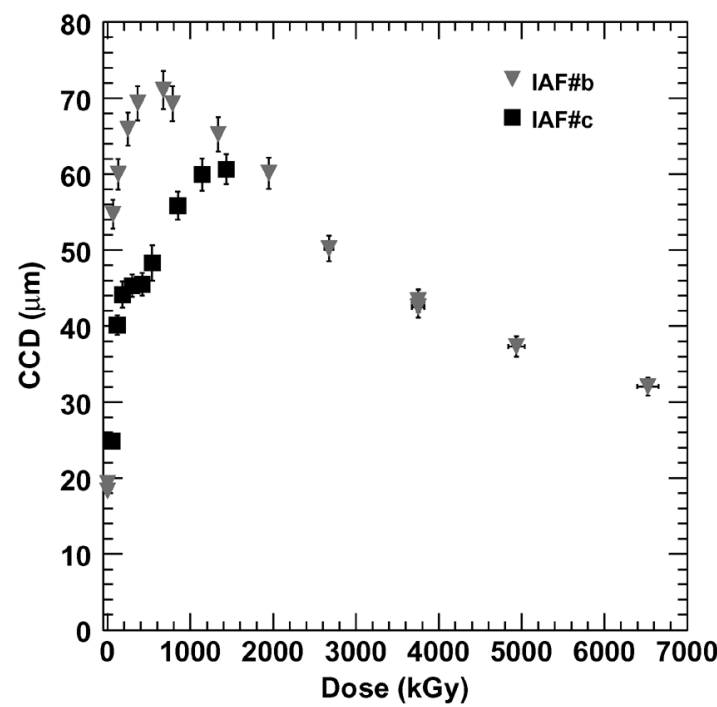

Fig. 9. The response of polycrystalline diamond sensors IAF as a function of the absorbed dose from $10 \mathrm{MeV}$ electrons. The bias voltage applied to the sensors was $400 \mathrm{~V}$. (IAF samples, $500 \mu \mathrm{m}$ thick, $0.8 \mathrm{~V} / \mu \mathrm{m}$ ).

achieved a maximal absorbed dose of about 7 MGy for one sensor from each manufacturer. A second sample from each manufacturer was irradiated to about $1.5 \mathrm{MGy}$. The response to particles from a ${ }^{90} \mathrm{Sr}$ was measured in intervals of about one hour while keeping the bias voltage applied to the sensor continuously. The sensors show a clear increase of the CCD at the beginning of the irradiation. Such a dependence on the absorbed dose was the first time discussed in [12]. In this period the existing trapping centers in the crystal are filled with charge carriers and do not contribute any longer to the signal degradation. The CCD therefore increases, a phenomenon denoted hereafter as pumping. As we continue the irradiation we observe a decrease of the CCD in most cases. The two samples irradiated only to about $1.5 \mathrm{MGy}$ just enter this phase of degradation and the sensor IAF\#c has apparently reached the point of 


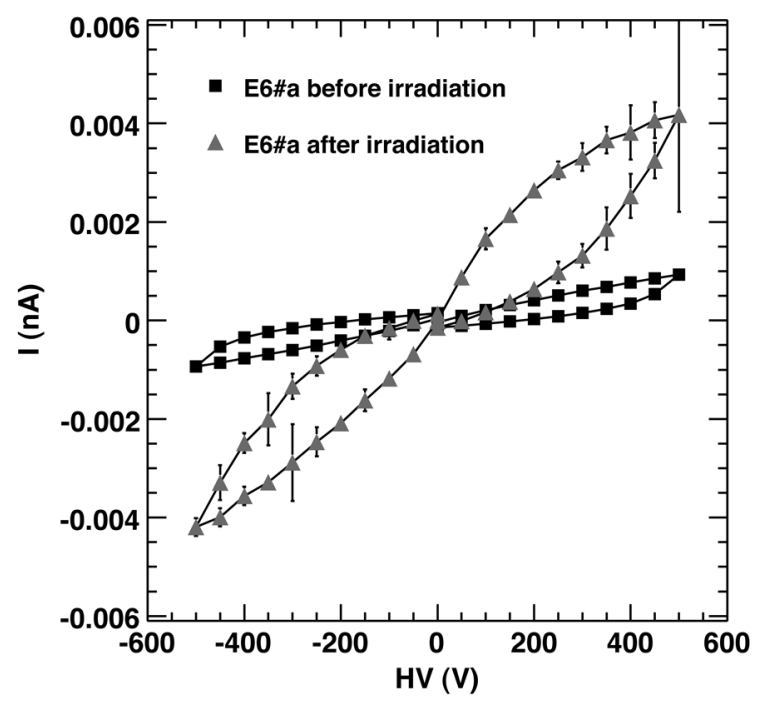

Fig. 10. Current-voltage characteristics before and after irradiation of one of the E6 samples. Higher absolute currents were observed during the ramping-up phase of the voltage.

maximal pumping. The sensors irradiated up to $7 \mathrm{MGy}$ show a very significant decrease of the $\mathrm{CCD}$, albeit the absolute value of CCD after $7 \mathrm{MGy}$ is still at the level of the initial CCD before pumping. All sensors are functional after the irradiation.

\section{B. Properties Before and After Irradiation}

All irradiated sensors are tested in the laboratory for changes in their current-voltage characteristics or in the dependence of the CCD on the applied electric field. Between irradiation and the measurements the samples were stored for approximately one week at room temperature avoiding any illumination. Fig. 10 shows as an example a comparison of the current-voltage measurement of the sensor E6\#a. Albeit a slight increase of the current is clearly visible, the current is still of the order of a few pA and is uncritical for operating the diamond as a detector. The hysteresis during the measurement is a known effect in pCVD diamond and is due to polarization effects. The results for the other sensors are similar.

The CCD as a function of the applied electric field shows a decrease for most of the samples as can be seen in Fig. 11, where a measurement for the sensor E6\#a is shown. This sensor was irradiated up to about $7 \mathrm{MGy}$. The CCD decreases by approximately $80 \%$ compared to the measurement before irradiation. Note that the sensors are fully depumped in both cases by the use of UV illumination for several minutes. It was observed that by pumping the sensor with a dose of about $20 \mathrm{~Gy}$ the CCD recovers partly. This change in the pumping behavior indicates the creation of shallow trapping centers in the crystal by the irradiation with $10 \mathrm{MeV}$ electrons.

\section{Comparison With Silicon}

A standard p-n silicon sensor is irradiated under the same conditions as described above. The bulk material is high ohmic silicon in 111 orientation with a resistivity of $3-5 \mathrm{k} \Omega \mathrm{cm}$.

We did not cool or modify the shaping time of the preamplifier during our measurements even if these actions could have

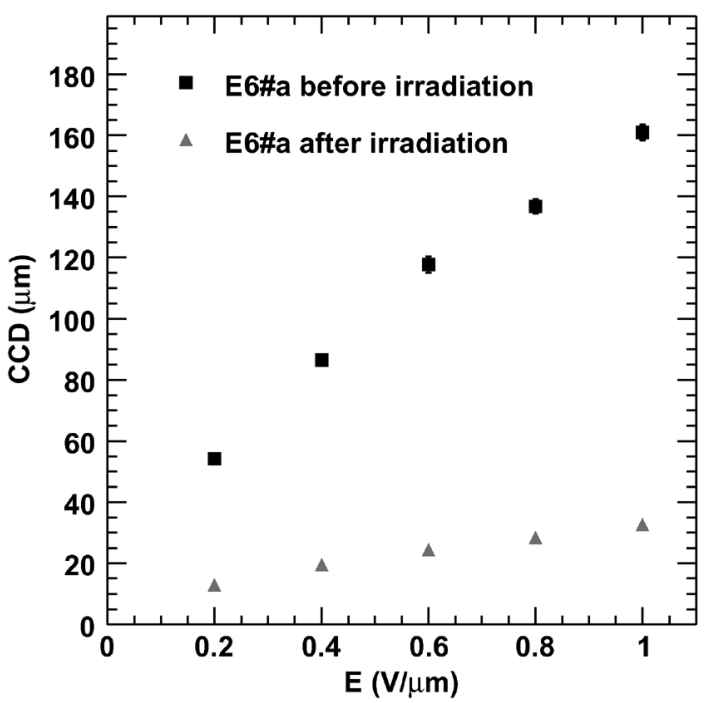

Fig. 11. CCD of E6\#a as a function of the applied electric field compared between before and after irradiation.

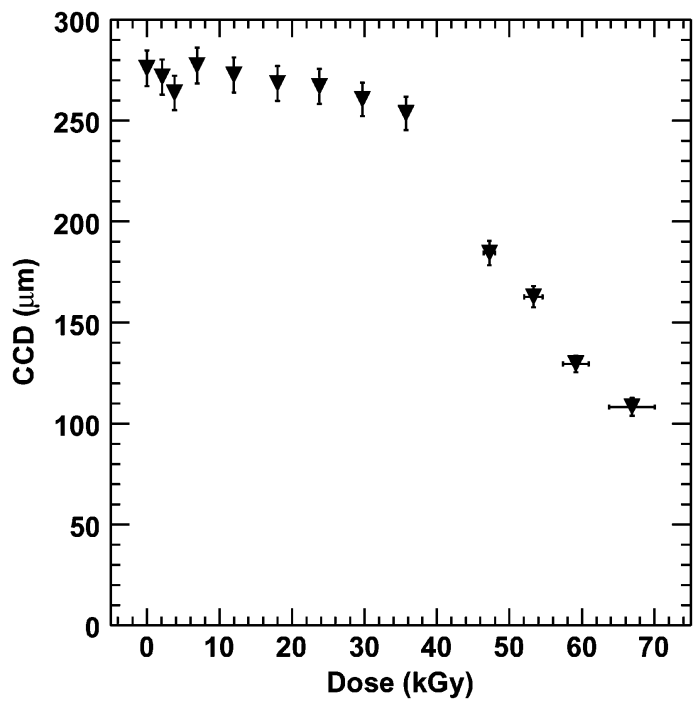

Fig. 12. CCD in dependence of the absorbed dose for a standard silicon sensor. The sensor thickness is $280 \mu \mathrm{m}$. The bias voltage applied to the sensor was $50 \mathrm{~V}$.

improved the performance of the measurement. The dose rate is set to the lower limit of the accelerator facility which is at about $1 \mathrm{nA}$ beam current. To facilitate the comparison the silicon sensor efficiency is also given in terms of the CCD, which is equal to the sensor thickness of $280 \mu \mathrm{m}$ at the start of the irradiation. Fig. 12 shows the effect of the irradiation. The CCD of the silicon sensor starts to decrease after absorbing about $35 \mathrm{kGy}$. The leakage current of the sensor increases during the irradiation from the level of $1 \mathrm{nA}$ at $50 \mathrm{~V}$ to more than $6 \mu \mathrm{A}$.

\section{CONCLUSION}

We investigated polycrystalline CVD diamond sensors for the application as sensor in the BeamCal of the ILC detectors. The linearity of the response of pCVD diamond sensors up to a particle flux of $5 \times 10^{6} \mathrm{MIP} /\left(\mathrm{cm}^{2} \times 10 \mathrm{~ns}\right)$ was shown to be within $30 \%$, which is of the order of the systematic uncertainty of the flux calibration. We investigated the radiation hardness 
with $10 \mathrm{MeV}$ electrons. It was shown that the diamond sensors are still operational after absorbing up to about $7 \mathrm{MGy}$. A loss in the charge collection efficiency due to this irradiation was observed proving the creation of defects in the crystal lattice. It is possible to partially recover the sensor CCD by pumping the samples with low doses of several Gy.

\section{ACKNOWLEDGMENT}

The authors would like to thank the CERN PS and S-DALINAC operators for their great support during the test beam periods. The authors would also like to thank The International Association for the Promotion of Co-operation with Scientists from the New Independent States of the Former Soviet Union (INTAS).

\section{REFERENCES}

[1] H. Abramowicz, K. Afanaciev, S. Denisov, R. Dollan, D. Drachenberg, and V. Drugakov et al., "Instrumentation of the very forward region of a linear collider detector," IEEE Trans. Nucl. Sci., vol. 51, no. 6, pp. 2983-2989, Dec. 2004.

[2] LDC Working Group, The LDC Outline Document [Online]. Available: http://www.ilcldc.org

[3] S. Agostinelli, J. Allison, K. Amako, J. Apostolakis, H. Araujo, and P. Arce et al., "GEANT4: A simulation toolkit," Nucl. Instrum. Methods Phys. Res. A, vol. A506, pp. 250-303, 2003, GEANT4 Collaboration.
[4] T. Raubenheimer, ILC Suggested Beam Parameters Range [Online]. Available: http://www-project.slac.stanford.edu/ilc/acceldev/beamparameters.html

[5] M. H. Nazare, B. Foster, R. S. Gilmore, T. J. Llewellyn, R. J. Tapper, and S. Roe et al., "Development of Diamond Tracking Detectors for High Luminosity Experiments at the LHC," CERN-DRDC-94-21, DRDC-P-56, Geneva, RD 42, 1994.

[6] W. Adam, W. de Boer, E. Borchi, M. Bruzzi, C. Colledani, and P. D. Angelo, "Radiation hard diamond sensors for future tracking applications," Nucl. Instrum. Methods Phys. Res. A, vol. A565, pp. 278-283, 2006.

[7] T. Behnke, M. Doucet, N. Ghodbane, and A. Imhof, "Radiation hardness and linearity studies of CVD diamonds," Nucl. Phys. Proc. Suppl., vol. 125 , pp. $263-266,2003$

[8] G. Lutz, Semiconductor Radiation Detectors-Device Physics. Berlind, Germany: Springer, 1999, ISBN 3-540-64-859.

[9] S. Zhao, "Characterization of the Electrical Properties of Polycrystalline Diamond Films," Ph.D. dissertation, Ohio State Univ., Columbus, 1994.

[10] D. Chong, L. Fernandez-Hernando, R. Gray, C. J. Ilgner, A. L. Macpherson, and A. Oh et al., "Validation of synthetic diamond for a beam condition monitor for the compact muon solenoid experiment," in Proc. IEEE Nuclear Science Symp. Conf. Rec., 2004, vol. 3, pp. $1812-1815$.

[11] E. Kuznetsova, "Design Studies and Sensor Tests for the Beam Calorimeter of the ILC Detector," Ph.D. dissertation, Humboldt University, Berlin, Germany, 2005.

[12] C. Bauer, I. Baumann, C. Colledani, J. Conway, P. Delpierre, and F. Djama et al., "Recent results from the RD42 diamond detector collaboration," Nucl. Instrum. Methods Phys. Res. A, vol. A383, pp. 64-74, 1996. 